\title{
Evaluación de la Polarización óptica en imágenes
}

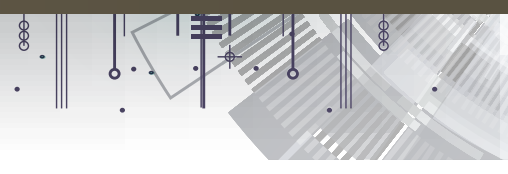

Jorge Benalcázar ${ }^{1}$, Fabián Cuzme ${ }^{1}$

${ }^{1}$ Universidad Técnica del Norte, Facultad de Ingeniería en Ciencias Aplicadas, Carrera en

Telecomunicaciones, Av. 17 de Julio y General José María Córdova, Ibarra, Ecuador

1jrbenalcazar@utn.edu.ec, ${ }^{1}$ fgcuzme@utn.edu.ec

\section{RESUMEN}

Para entender mejor la incidencia que tiene la óptica ondulatoria al momento de captar electrónicamente la imagen formada detrás de una lente, es necesario establecer una base científica en lo referente a polarización óptica, este fundamento científico-técnico también nos sugiere la metodología a seguir. Optamos por el método experimental, se procede a diseñar y construir el montaje óptico para generar la imagen de un objeto planar cercano, se refleja en una superficie dieléctrica, es filtrada con un polaroide analizador para ser fotografiada y almacenada, la aparición de patrones de cambio en dichas imágenes es explicable con la evaluación del grado de polarización de sus partes, mediante algoritmos y programas. Se establece criterios de comparación que permiten tener versatilidad al momento de procesar las imágenes obtenidas, MATLAB es una de ellas y ayudó a desarrollar un algoritmo propio para binarización, segmentación, transformación y comparación de las imágenes, esto permite conocer los índices de diferencias que existen entre ellas, este resultado se expresa mediante subfotogramas de cambio, mismos que demuestran una pérdida de nitidez por efecto de la polarización.

Palabras Claves: Grado de polarización, segmentación de imagen, algoritmos de comparación, imágenes binarias.

\section{ABSTRACT}

A scientific base is established on the topics of: linear, circular and elliptical polarization, Poincaré sphere, Jones and Stokes vectors and Moeller matrices. This scientific foundation helps in understanding the phenomenon and also indicates the size of the task, as well as the optical assembly to design and build. The image of a nearby planar object lights up and reflected from a dielectric surface, filtered with an analyzer polaroid to be captured by a CCD, and stored. The evaluation of the changes in said image is subject to the analysis of the degree of polarization of its parts through algorithms and constitutes the objective of this work. The importance of knowing adequate tools for the acquisition and analysis of images is fundamental, so it was necessary to establish comparison criteria that allow greater versatility when processing the images obtained, MATLAB is one of them, it helps to develop its own algorithm for binarization, segmentation, transformation and comparison of the images, this allows to know the indices of differences that exist between them, this result is expressed as a change matrix.

Keywords: Degree of polarization, image segmentation, comparison algorithms, binary images. 


\section{Introducción}

La detección de reflejos en las microfibras de telas es testigo de la presencia de algún porcentaje de material sintético en las mismas y constituye el apoyo de la imagenología polarimétrica a los métodos de evaluación de calidad de los tejidos (las fibras y telas), en la medicina se puede identificar patrones de cambio del estado de polarización de la piel asociados a razones clínicas. En cualquier caso, se hace necesaria la utilización de un sistema de cámara fotográfica o de TV con lentes acromáticos y un sistema de almacenamiento.

La polarización de la luz brinda patrones específicos para la reflexión de imágenes desde diversas superficies dieléctricas, el entendimiento de dichos patrones y su procesamiento digital permitirán analizar las imágenes provenientes de superficies como: parabrisas de autos o ventanas de edificaciones, se abre la posibilidad de detección del vidrio templado o polarizado con el posible análisis de su calidad, aplicación en actividades agrícolas, industriales y/o medicinales. En el presente trabajo se logra demostrar una relación entre los patrones de las imágenes reflejadas en dieléctricos y la polarización óptica.

Recordemos la conocida expresión algebraica de óptica geométrica para la posición de la imagen de un punto detrás de un lente: $\quad \frac{1}{o}+\frac{1}{i}=\frac{1}{f}$

o.- es la distancia desde el objeto luminoso hasta el vértice del lente, i.- es la distancia medida desde el lente hasta el plano donde se forma la imagen y $f$ es la distancia focal del lente [1], figura No. 1 


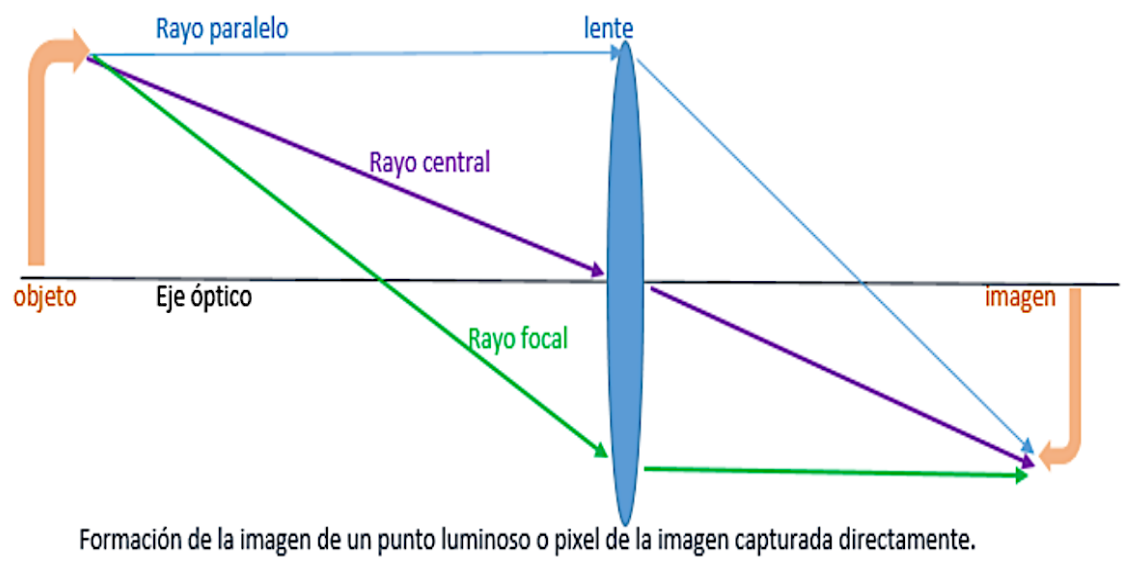

Fig. 1. Formación de la imagen de un punto luminoso detrás de una lente convergente.

La fórmula (1) en óptica geométrica, determina la posición del punto luminoso imagen, pero no considera el factor ondulatorio de polarización de los rayos en el trayecto hacia el censor, cuando se graba el reflejo cercano de un objeto luminoso, y se utiliza un esquema configurado como lo indica la figura 2, cada punto del objeto constituye una mini fuente de iluminación y posee su propia función de distribución angular al igual que su propia polarización. Se conoce poco acerca de la composición polarimétrica de cada rayo.

La luz viajará hasta el CCD de la cámara, donde $p$ es la distancia desde el objeto luminoso hasta el lente, q es la distancia imagen medida desde el lente hasta el CCD y f es la distancia focal del lente, en su camino se polarizará por reflexión [2], al final es captada por alguno de los censores fotosensibles del CCD y caracterizado por las coordenadas $\mathrm{x}$ e y en el microchip, entonces se convierte en señal eléctrica, de ésta forma se constituye como un pixel, a partir de éste momento la información estará condicionada al formato del de imagen de la cámara [3], [4].

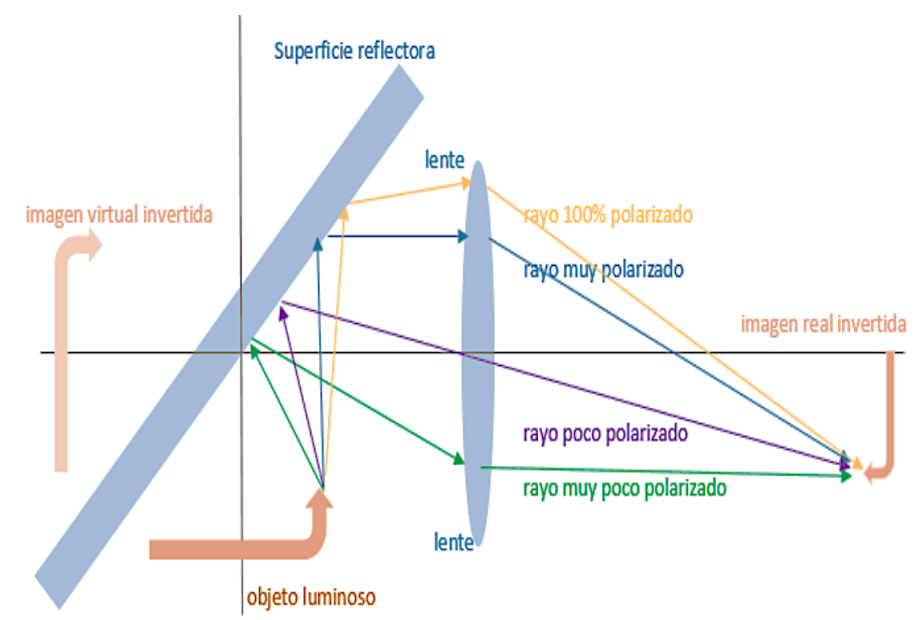

Formación de la imagen de un punto luminoso o pixel de la imagen reflejada y luego capturada.

Fig. 2. Polarización de los rayos reflejados que convergen para formar la imagen. 
El análisis experimental de dicho punto luminoso considerando la polarización, requeriría de varios pasos:

- Que cada rayo no se refleje, no se esparza ni se filtre, es decir que captaremos la imagen original de un punto luminoso del objeto, el mismo razonamiento y análisis podemos aplicar a todos y cada uno de los puntos luminosos, obtenemos entonces la imagen virgen de un grupo de puntos luminosos (antes).

- Si cada rayo se refleja antes de ser detectado, entonces tendremos la imagen del mismo punto luminoso, pero con alteración inducida en su polarización [5], cada uno de los rayos aporta a la formación de un pixel, en consecuencia, capturamos pixel por pixel la imagen completa del objeto luminoso (después).

- Solo es posible separar la acción de luz polarizada del efecto del resto de rayos con la presencia de un polaroid analizador, éste filtra por lo menos parcialmente la luz polarizada, de manera que se puede tener $I_{\max }$ e $I_{\min }$ para cada pixel y luego evaluar la polarización de la imagen con la utilización de la fórmula:

$$
p=\frac{I_{\max }-I_{\min }}{I_{\max }+I_{\min }}
$$

[6], [7], válida para rayos paraxiales [8].

- Existe la posibilidad de asignar un valor $p$ específico a todos rayos polarizados y un valor $p$ diferente a los no polarizados, de ésta manera quedaría catalogado cada rayo y su respectivo pixel, con los valores de p se puede construir la matriz de polarización. Sin embargo éste análisis no se ejecuta ni se incluye en el presente trabajo.

- En el caso de objetos reflejados desde muy lejos la llegada al lente de la cámara se da con rayos paraxiales, tendríamos poca fluctuación en lo referente a ángulos, también menos rayos y pixeles para cada sector de la imagen, ante la rotación acimutal del analizador, la intensidad de éstas imágenes cambia de acuerdo a las leyes de Fresnel y en ciertos casos prácticamente desaparecerían.

- Está presente la opción de fragmentar la imagen en grupos de píxeles de tamaños definidos y analizar la luminancia de cada grupo por separado considerando el cambio por umbralización en tonos de gris [9], es decir utilizando una matriz $\mathrm{m} \times \mathrm{n}$ que en lo futuro llamaremos matriz de fragmentación.

Considerando todos estos factores, el documento se concatena como sigue:

Con el propósito de analizar la influencia ondulatoria de la polarización en imágenes reflejadas desde cerca, se plantea la base científica en el campo de polarización óptica, vectores de Jones y de Stokes, Matrices de Moeller y esfera de Poincaré, se presenta algunos conceptos específicos necesarios para entender y describir el fenómeno, definir el camino experimental y estimar el tamaño de la tarea.

En la sección de materiales y métodos, se diseña, construye y prueba el montaje óptico acorde a la arquitectura descrita en la figura 8 , su funcionamiento exitoso constituye el primer resultado experimental, el cual permite la generación de imágenes parcialmente polarizadas durante la reflexión y su posterior captura fotográfica ya con diversos índices de polarización inducida por un polaroide analizador de rotador graduado. 
En la Sección de resultados, se complementa con los algoritmos y programas que constituyen el segundo resultado, se juntan para ser mostrados al final con el patrón de transformación de imagen, el cual se expresa mediante subfotogramas (se los llama figuras $11,12,13$ y 14). Además, los datos cuantitativos y cualitativos se comentan en la sección de resultados.

\section{Base Científica}

Aquí se revisan las definiciones de polarización lineal, circular y elíptica; se lo hace de cuatro formas: forma gráfica, algebraica como función de onda, a cada una se le asigna una posición específica sobre la superficie de la esfera de Poincaré y finalmente se explica brevemente el comportamiento de la luz. El lector debe prestar atención a los colores de la letra con que se escribe algunas expresiones y funciones, con los mismos tonos aparecen los planos de polarización en la figura 7.

Para una mejor comprensión del tema, se explicará los vectores de Jones y se adjunta fotos de maquetas de las ondas, la ecuación de la cónica característica, y finalmente se asigna un gráfico y un vector posición sobre la esfera de Poincaré y la respectiva elipse.

\section{Polarización lineal}

Las ondas electromagnéticas se caracteriza por la perpendicularidad entre los vectores $\vec{E}, \vec{H}$ y $\overrightarrow{\pi_{r}}$, siendo la polarización un efecto característico de las ondas transversales, una onda se denomina polarizada linealmente o también "onda planarmente polarizada" o linealmente polarizada, aquella para la cual, el campo electrostático todo el tiempo está en el mismo plano como se ilustra a continuación:
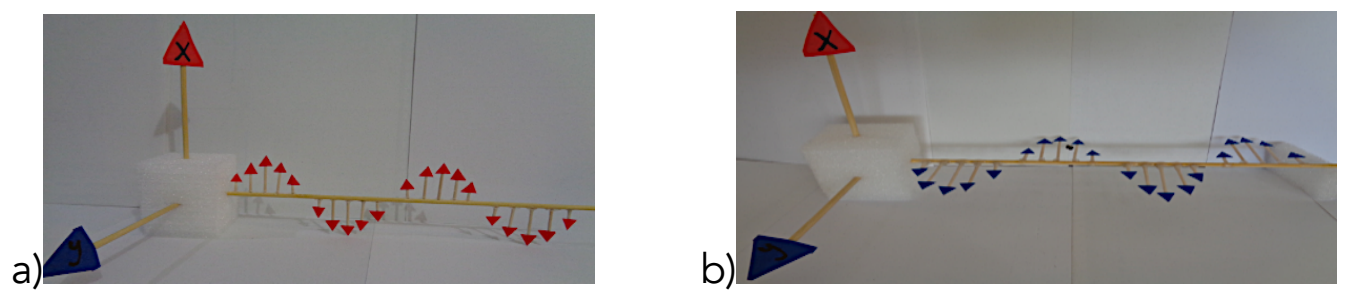

Fig.3. Foto de "ondas esquemáticas" polarizadas linealmente; a) En el plano rx (vectores rojos), b) En el plano ry(vectores azules)

En la figura 3a), al plano xr se lo denomina "plano de oscilación" debido a que en dicho plano oscila el campo electrostático de la onda representada con rojo. Diremos entonces que la onda está polarizada en el plano horizontal xr, para esta onda tenemos la expresión algebraica:

$E_{x}(r, t)=E_{o x} \cos \left\{\frac{2 \pi r}{\lambda} \mp \frac{2 \pi t}{T}\right\}(3)$, simbolizada por el vector; $(+1,0,0)$ sobre la esfera de Poincaré (figura 6) [10]. 
En Ias tiguras SD), se visuaııza el piano ae oscilación" debido a que en dichos planos oscila el campo eléctrico de las ondas. Diremos entonces que la onda está polarizada en el plano vertical yr, para esta onda tenemos la expresión:

$$
E_{y}(r, t)=E_{o y} \cos \left\{\frac{2 \pi r}{\lambda} \mp \frac{2 \pi t}{T}\right\}(4) \text { se representa la onda con el vector; }(-1,0,0) \text { sobre }
$$
la misma esfera (figura 6).

A $90^{\circ}$ en lo que sería el eje y, está la polarización diagonal en el plano inclinado $+45^{\circ}$ sobre el ecuador de la esfera, es decir cuando $E_{o x}=E_{o y}$ : $\left[\begin{array}{l}E_{x}(r, t)=E_{o x} \cos \left\{\frac{2 \pi r}{\lambda} \mp \frac{2 \pi t}{T}\right\} \\ E_{y}(r, t)=E_{o y} \cos \left\{\frac{2 \pi r}{\lambda} \mp \frac{2 \pi t}{T}\right\}\end{array}\right]$ (5) aquí se usa el vector $(0,1,0)$ en el ecuador.

A $270^{\circ}$ en lo que sería el eje -y, está la polarización diagonal en el plano inclinado $-45^{\circ}$ bajo el ecuador, esto es cuando $\quad E_{o x}=-E_{o y}:\left[\begin{array}{c}E_{x}(r, t)=E_{o x} \cos \left\{\frac{2 \pi r}{\lambda} \mp \frac{2 \pi t}{T}\right\} \\ E_{y}(r, t)=-E_{o x} \cos \left\{\frac{2 \pi r}{\lambda} \mp \frac{2 \pi t}{T}\right\}\end{array}\right]$, (6) tenemos como referencia el vector; $(0,-1,0)$ sobre la línea ecuatorial.

Los otros estados de polarización lineal diagonal se representa en la figura 7b.

Los rayos con campo paralelo al eje óptico pasan casi sin ser absorbidos, de esta forma la mitad de los rayos absorbidos tienen su campo eléctrico perpendicular a los rayos que atraviesan, es decir que los que atraviesan tienen el mismo grado de polarización, entre ellos se obtiene luz linealmente polarizada en el plano paralelo al eje óptico [11].

Otra forma de obtener luz polarizada en forma experimental es hacer reflejar luz natural en un cristal común bajo el Ángulo de Brewster, cuando el rayo reflejado es perpendicular al rayo refractado se obtiene luz $100 \%$ polarizada en el plano perpendicular al plano de reflexión, en este caso la intensidad luminosa reflejada es muy pequeña [12].

\section{Polarización circular}

Si se superpone dos campos eléctricos perpendiculares de la misma magnitud y periodicidad pero desfasados un ángulo $\delta \equiv-\pi / 2$ como los que se ilustra en los gráficos a) y b) tendremos el caso:

conduce a la expresión:

$$
\left[\begin{array}{c}
E_{x}(r, t)=E_{o x} \cos \left\{\frac{2 \pi r}{\lambda}-\frac{2 \pi t}{T}\right\} \\
E_{y}(r, t)=E_{o y} \cos \left\{\frac{2 \pi r}{\lambda}-\frac{2 \pi t}{T}+\delta\right\}
\end{array}\right] \text { (7), una breve transformación nos }
$$

$$
\left[\begin{array}{l}
E_{x}(r, t)=E_{o} \cos \left\{\frac{2 \pi r}{\lambda} \mp \frac{2 \pi t}{T}\right\} \\
E_{y}(r, t)=E_{o} \operatorname{sen}\left\{\frac{2 \pi r}{\lambda} \mp \frac{2 \pi t}{T}\right\}
\end{array}\right] \text { (8), tenemos la ecuación de una circunferencia de radio }
$$


constante $E_{o}$, de ahí el nombre. Dicho radio gira alrededor del eje $r$ a medida que la onda avanza a lo largo de $r$ de acuerdo a la ley de la mano derecha en sentido horario como se visualiza en las figuras $4,6 b$ y $7 a$ :

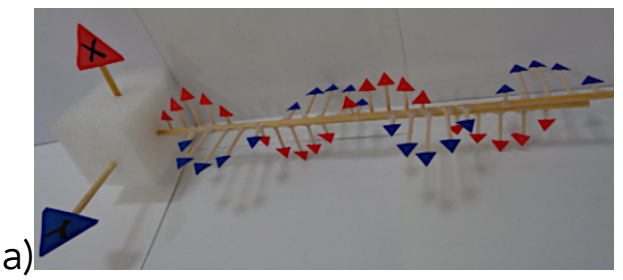

b)

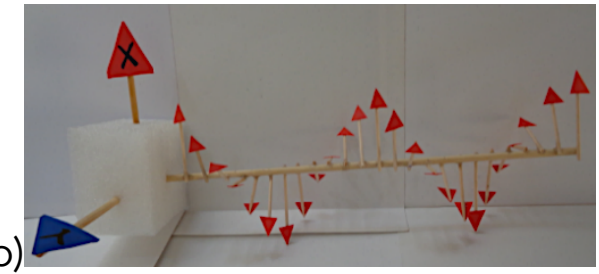

Fig. 4. a) Formación de una onda circular derecha b) Onda circular derecha formada.

A esta circunferencia se le asigna el vector $(0,0,1)$ que no es sino el eje $+z$, es decir el polo norte de las figuras No. 6 y 7 a.

De manera similar si $\delta \equiv+\pi / 2$, el radio será $E_{o}$ y gira alrededor de $r$ en sentido antihorario a medida que la onda avanza;

$\left[\begin{array}{c}E_{x}(r, t)=E_{o} \cos \left\{\frac{2 \pi r}{\lambda} \mp \frac{2 \pi t}{T}\right\} \\ E_{y}(r, t)=-E_{o} \operatorname{sen}\left\{\frac{2 \pi r}{\lambda} \mp \frac{2 \pi t}{T}\right\}\end{array}\right](9)$, a esta circunferencia le asignaremos el vector

$(\mathbf{0}, \mathbf{0},-\mathbf{1})$ en el equivalente al eje $-\mathrm{z}$, tal como se visualiza en el polo sur de las figuras 6 y $7 a$.

Cuando un rayo polarizado circular derecho atraviesa un polaroid, éste absorberá en mayor grado el campo en una dirección y en mucho menor en la dirección perpendicular, es decir que obtendremos luz linealmente polarizada. Lo mismo sucederá con un haz de rayos polarizados circulares izquierdas atraviese una placa de turmalina o un polaroid. [13].

\section{Polarización elíptica}

Si se superpone dos campos eléctricos perpendiculares de diferente magnitud y la misma periodicidad pero desfasados un ángulo $\delta$ como los que se ilustra en los gráficos $7 a$ y $7 b$
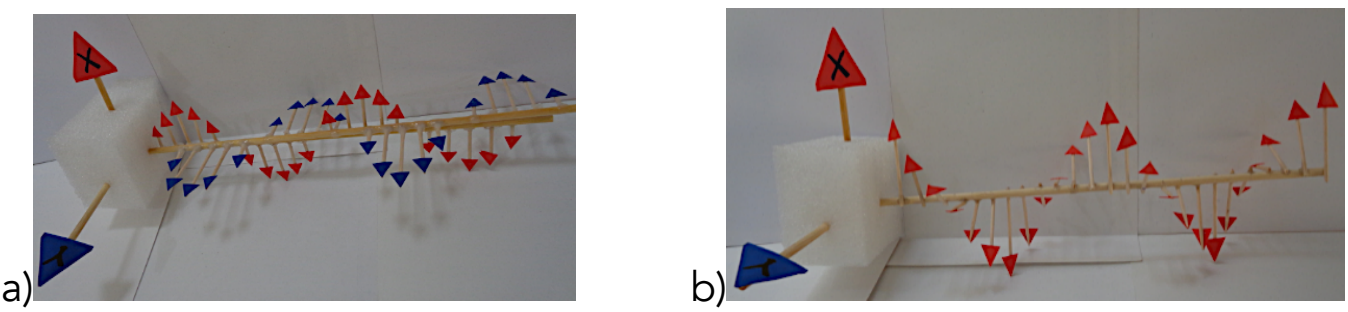

Fig.5. a) Formación de una onda elíptica derecha, b) Onda elíptica derecha formada. 
Con el mismo desarrollo algebraico se llega a una elipse, el vector de campo E rota a la izquierda mientras avanza en $r$ describiendo dicha cónica. Para los casos en que $\delta \neq$ $\pm \pi / 2$ se tendrán elipses inclinadas con diferentes excentricidades, cada una de ellas encontrará un punto en la esfera de Poincaré que la describa adecuadamente. Tendremos el caso:

$$
\left[\begin{array}{c}
E_{x}(r, t)=E_{o x} \cos \left\{\frac{2 \pi r}{\lambda}-\frac{2 \pi t}{T}\right\} \\
E_{y}(r, t)=E_{o y} \cos \left\{\frac{2 \pi r}{\lambda}-\frac{2 \pi t}{T}-\delta\right\}
\end{array}\right]
$$

si $\delta \equiv-\pi / 2$, entonces con un breve desarrollo algebraico llegamos a la ecuación de la elipse de donde toma su nombre.

A $0^{\circ}$ sobre el meridiano que pasa por el eje $x$, está representada la polarización elíptica horizontal, es decir cuando $E_{o x}=\epsilon E_{o y}=E_{o} \quad$, algebraicamente tenemos:

$$
\left[\begin{array}{l}
E_{x}(r, t)=\epsilon E_{o} \cos \left\{\frac{2 \pi r}{\lambda} \mp \frac{2 \pi t}{T}\right\} \\
E_{y}(r, t)=E_{o} \operatorname{sen}\left\{\frac{2 \pi r}{\lambda} \mp \frac{2 \pi t}{T}\right\}
\end{array}\right]
$$

usaríamos el vector unitario; $\left(\cos \frac{90^{\circ}}{\epsilon}, 0, \operatorname{sen} \frac{90^{\circ}}{\epsilon}\right)$ para elipses horarias o levógiras en el hemisferio Norte, mientras que $\left(\cos \frac{90^{\circ}}{\epsilon}, 0,-\operatorname{sen} \frac{90^{\circ}}{\epsilon}\right)$ para elipses dextrógiras $u$ antihorarias en el hemisferio Sur.

A los $180^{\circ}$ sobre el meridiano que pasa por el eje -x, está representada la polarización elíptica vertical, esto es cuando $E_{o y}=\vartheta E_{o x}=E_{o}$ y tenemos:

$$
\left[\begin{array}{c}
E_{x}(r, t)=E_{o} \cos \left\{\frac{2 \pi r}{\lambda} \mp \frac{2 \pi t}{T}\right\} \\
E_{y}(r, t)=\vartheta E_{o} \operatorname{sen}\left\{\frac{2 \pi r}{\lambda} \mp \frac{2 \pi t}{T}\right\}
\end{array}\right]
$$

tomamos entonces el vector; $\left(-\cos \frac{90^{\circ}}{\vartheta}, 0, \operatorname{sen} \frac{90^{\circ}}{\vartheta}\right)$ para elipses horarias o levógiras en el hemisferio Norte, mientras que $\left(\cos \frac{90^{\circ}}{\vartheta}, 0,-\operatorname{sen} \frac{90^{\circ}}{\vartheta}\right)$ para elipses dextrógiras $u$ antihorarias en el hemisferio Sur.

A $90^{\circ}$ sobre el meridiano que pasa por el eje y, está la polarización elíptica diagonal levógira u horaria con el eje mayor inclinado $45^{\circ}$ respecto a la línea ecuatorial, es decir cuando $E_{o x}=\epsilon E_{o y}=E_{o}$

$$
\left[\begin{array}{l}
E_{x}(r, t)=E_{o x} \cos \left\{\frac{2 \pi r}{\lambda} \mp \frac{2 \pi t}{T}\right\} \\
E_{y}(r, t)=E_{o y} \operatorname{sen}\left\{\frac{2 \pi r}{\lambda} \mp \frac{2 \pi t}{T}\right\}
\end{array}\right]
$$


entonces usamos el vector; $\left(0, \cos \frac{90^{\circ}}{\epsilon}, \operatorname{sen} \frac{90^{\circ}}{\epsilon}\right)$ para elipses horarias o levógiras en el hemisferio Norte, mientras que $\left(0, \cos \frac{90^{\circ}}{\epsilon},-\operatorname{sen} \frac{90^{\circ}}{\epsilon}\right)$ para elipses dextrógiras $u$ antihorarias en el hemisferio Sur.

A $270^{\circ}$ sobre el meridiano que pasa por el eje -y, está la polarización elíptica diagonal levógira u horaria con el eje mayor inclinado $135^{\circ}$ respecto a la línea ecuatorial, es decir cuando $E_{o x}=\epsilon E_{o y}=E_{o}$

$$
\left[\begin{array}{l}
E_{x}(r, t)=E_{o x} \cos \left\{\frac{2 \pi r}{\lambda} \mp \frac{2 \pi t}{T}\right\} \\
E_{y}(r, t)=E_{o y} \operatorname{sen}\left\{\frac{2 \pi r}{\lambda} \mp \frac{2 \pi t}{T}\right\}
\end{array}\right]
$$

entonces usamos el vector; $\left(0,-\cos \frac{90^{\circ}}{\epsilon}, \operatorname{sen} \frac{90^{\circ}}{\epsilon}\right)$ para elipses antihorarias o dextrógiras en el hemisferio Norte, mientras que $\left(0,-\cos \frac{90^{\circ}}{\epsilon},-\operatorname{sen} \frac{90^{\circ}}{\epsilon}\right)$ para elipses dextrógiras o antihorarias en el hemisferio Sur, se ilustra en la figura 6 a y b.

\section{Esfera de Poincaré}

La Esfera de Poincaré constituye un mapa esférico donde se pueden describir todos los estados de polarización posibles, desde la polarización circular en los polos hasta la lineal en el ecuador como se representa en las siguientes figuras:
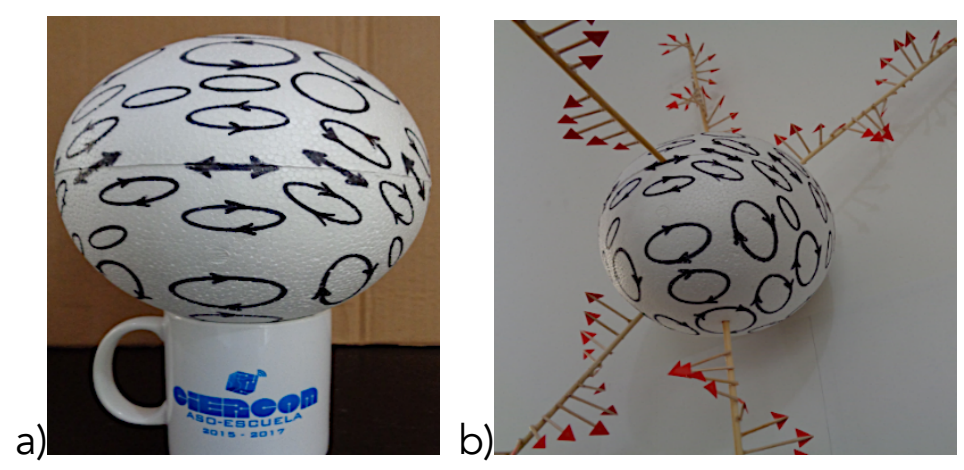

Fig.6. a) Esfera esquemática de Poincaré, b)Esfera de Poincaré con las respectivas ondas.

Sobre la superficie de una esfera de espumaflex fueron dibujadas algunas elipses, en ellas se representa el tipo de polarización, no se conservó circulación de mano derecha (la ley de la mano derecha). En los sitios correspondientes, se incrustaron "ondas esquemáticas" construidas en madera y cartón, así visualizaremos el tipo de onda en cuestión.

- $\quad$ N.- El hemisferio norte de la esfera representa la polarización levógira (de mano izquierda u horaria), El polo norte $\mathrm{N}$ en si será: Right Circular Polarization (RCP).

- S.- El hemisferio sur de la esfera representa la polarización circular dextrógira (de mano derecha o antihoraria), El polo Sur en si será: Left Circular Polarization (LCP) [14]. Los dos casos pueden visualizarse en el siguiente gráfico: 
a)

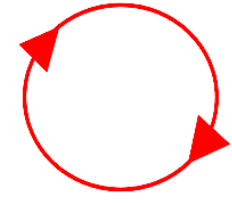

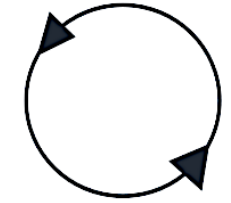

Left Circular Polarization (LCP)+

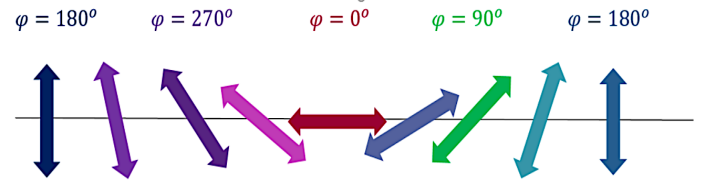

b) vertical $90^{\circ}$ diagonal $-45^{\circ}$ horizontal $0^{\circ}$ diagonal $45^{\circ}$ vertical $90^{\circ}$

Fig.7. Estados de polarización; a) Polos de la esfera, b) En la línea ecuatorial, $\varphi$ es la longitud en la esfera de Poincaré, al pie se indican algunas orientaciones notables del polaroide analizador.

- Sobre el ecuador se representa la Polarización lineal, a lo largo de los $360^{\circ}$ se indica la orientación de varios los planos de polarización sucesivos que se pueden encontrar.

De este modo el área que está entre los polos y el ecuador representa los diversos grados de la polarización elíptica tanto derecha como izquierda, se puede visualizar en la figura 6 a.

Como puede verse, la generalidad de los casos corresponde a polarización elíptica y solo en casos específicos tenemos lineal o circular. Un tubo de rayos de luz puede contener los tres tipos de polarización, se hace necesario definir de manera compacta su composición, esto se lo realiza mediante Vectores de Stokes, las matrices de Moeler contienen los cambios de estado de los vectores de Stokes.

\section{Vectores de Stokes y matrices de Moeller}

Una información más completa hace necesario el uso de un vector columna $\mathcal{S}$, que representa la composición o estado de polarización de la luz y usualmente se conoce como vector de Stokes

$$
\overrightarrow{\mathcal{S}^{\alpha}}=\left(\begin{array}{c}
s_{o}^{\alpha} \\
s_{1}^{\alpha} \\
s_{2}^{\alpha} \\
s_{3}^{\alpha}
\end{array}\right)=\left(\begin{array}{ccc}
\text { Iradiancia incidente } \\
\text { Pol.hor. } \mathbf{0}^{\boldsymbol{o}} ;+ & \text { ninguna } ; 0 & \text { Pol.vert. } \mathbf{9 0}^{\boldsymbol{o}} ;- \\
\text { Pol.diag. }+\mathbf{4 5}^{\boldsymbol{o}} ;+ & \text { ninguna } ; 0 & \text { Pol.obl. }-\mathbf{4 5}^{\boldsymbol{o}} ;- \\
\text { Circ.o elip. der } ;+ & \text { ninguna } ; 0 & \text { Circ.o elip. izq. } ;-
\end{array}\right)
$$

$\alpha=i$ indica que el el rayo incide, $\alpha=o$ cuando emerge del sistema [15].

$\mathcal{S}$ se define en función de las componentes ortogonales del vector de campo eléctrico (Ep,Es).

Para el caso de luz cuasi monocromática tendremos:

$$
\vec{E}(t)= \begin{cases}\vec{i} E_{o x} & \cos \left[(\bar{k} z-\bar{\omega} t)+\varepsilon_{x(t)}\right] \\ \vec{\jmath} E_{o y} & \operatorname{sen}\left[(\bar{k} z-\bar{\omega} t)+\varepsilon_{y(t)}\right] .\end{cases}
$$

Si además $\varepsilon_{x}-\varepsilon_{y}=\varepsilon$ y eliminamos la constante ${ }^{c \varepsilon_{o}} / 2$ para que los parámetros sean proporcionales a las irradiancias, entonces tendremos: 


$$
\left(\begin{array}{c}
s_{o}^{\alpha} \\
s_{1}^{\alpha} \\
s_{2}^{\alpha} \\
s_{3}^{\alpha}
\end{array}\right)=\left(\begin{array}{ccc}
\left\langle E_{o x}^{2}\right\rangle_{T} & + & \left\langle E_{o y}^{2}\right\rangle_{T} \\
\left\langle E_{o x}^{2}\right\rangle_{T} & - & \left\langle E_{o y}^{2}\right\rangle_{T} \\
\left\langle 2 E_{o x}\right. & E_{o y} & \cos \varepsilon\rangle_{T} \\
\left\langle 2 E_{o x}\right. & E_{o y} & \operatorname{sen} \varepsilon\rangle_{T}
\end{array}\right)
$$

La rotación acimutal del analizador intercalado delante del lente de acuerdo a la ley de Malus [15], hará disminuir significativamente la influencia de los rayos $100 \%$ polarizados y por lo tanto varía el grado de polarización p para dicho pixel, es decir cambia respuesta lineal del sistema óptico a un rayo de luz incidente y puede expresarse mediante la relación

$$
\overrightarrow{\mathcal{S}^{o}}=M \overrightarrow{\mathcal{S}^{\imath}}=\left(\begin{array}{llll}
m_{11} & m_{12} & m_{13} & m_{14} \\
m_{21} & m_{22} & m_{23} & m_{24} \\
m_{31} & m_{32} & m_{33} & m_{34} \\
m_{41} & m_{42} & m_{43} & m_{44}
\end{array}\right)\left(\begin{array}{c}
s_{o}^{i} \\
s_{1}^{i} \\
s_{2}^{i} \\
s_{3}^{i}
\end{array}\right)
$$

M es la matriz de Mueller del sistema, constituye una matriz de transformación cuadrada $4 \times 4$ de elementos reales [16], de esta manera cambió la composición de la polarización de un rayo reflejado. El análisis gráfico de sistemas polarimétricos puede realizarse utilizando representaciones en tres dimensiones (superficies) o en dos dimensiones (mapas), donde los respectivos ejes pueden estar asociados a los vectores de Stokes o bien a los ángulos elipsométricos asociados a los estados de polarización. Los vectores normalizados de Stokes pueden describirse gráficamente en término de los parámetros de la elipse de polarización, de los ángulos acimutal $(0 \leq \boldsymbol{\Psi} \leq \pi)$ y de elipticidad $(-\pi / 4 \leq \mathrm{X} \leq \pi / 4)$, respectivamente [17],

$$
\overrightarrow{\mathcal{S}}=\left\langle\mathcal{S}_{o}\right\rangle=\left(\begin{array}{c}
1 \\
\cos (2 \chi) \cos (2 \psi) \\
\cos (2 \chi) \operatorname{sen}(2 \psi) \\
\operatorname{sen}(2 \chi)
\end{array}\right)
$$

Donde $\left\langle\mathcal{S}_{o}\right\rangle$ representa la intensidad asociada al vector de Stokes, que usualmente se normaliza al valor unitario. [18].

El tamaño de ésta tarea supera la esfera del presente trabajo y nos abstenemos de ejecutar este análisis en función de las limitaciones.

\section{Materiales y Método}

En esta sección se establece una investigación práctica basada en la captación de imagen reflejada y filtrada con polaroid orientado a diferentes ángulos acimutales, así mismo una investigación descriptiva del fenómeno que permite analizar características observables aplicando el proceso de binarización y comparación de las imágenes captadas. Se diseña, construye y utiliza el montaje óptico necesario para la investigación. 


\section{Base Experimental}

En la figura 8 se representa la formación de un pixel rayo por rayo, todos provenientes de un mismo punto luminoso de la imagen reflejada en una placa del vidrio la cual juega el papel de polarizador [19], y por ende cada rayo se refleja con diferente grado de polarización, la única manera de conocer dicha magnitud es intercalar un rotador con polaroide analizador graduado para registrar imágenes cada cierto ángulo (10 o 15 grados) , es decir variando el ángulo acimutal con un rotador de polaroide graduado.

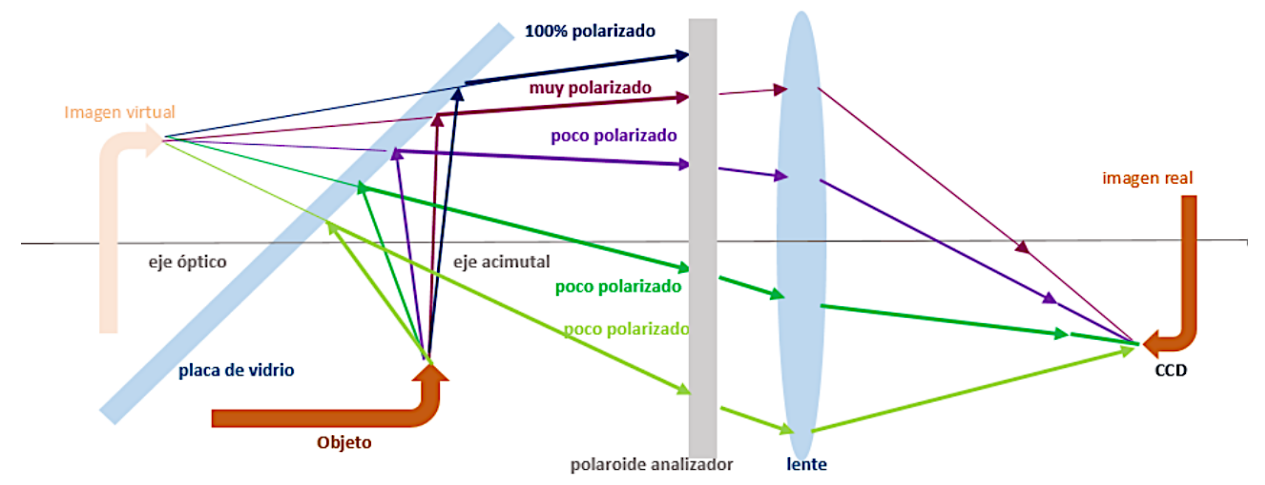

Fig.8. Formación de un pixel sobre el CCD de la cámara fotográfica

Los rayos que adquieren mayor grado de polarización son aquellos que se reflejan bajo ángulos cercanos al Ángulo de Brewster, cuando el eje permisivo del polaroide analizador este en concordancia con el plano perpendicular (posición $0^{\circ}$ ) dichos rayos pasarán libremente, pero al rotar el analizador de acuerdo a la Ley de Malus [20], ya no lo podrán atravesar, como consecuencia la intensidad luminosa sobre el CCD disminuye y la imagen se vuelve opaca y con tendencia al negro en la escala de grises, el cambio en el proceso de binarización es inminente.

Los puntos cercanos al Ángulo de Brewster tendrán grados de polarización altos, es entonces Heuristicamente razonable agrupar los puntos luminosos por su zona de procedencia ya que tendrán similar binarización, lo cual sugiere una Segmentación regular de Imagen por División del cuadro, ésto deja fuera los otros criterios de segmentación sugeridos [21]. 


\section{Procesamiento de imagen}

\section{Adquisición de las imágenes}

En la figura 8 el lente y el CCD corresponden a una cámara digital bajo la siguiente descripción:

Marca Sony Cyber-Shot modelo DSC-W830

CCD de 7,76 mm (tipo 1/2,3) filtro de color primario

Número efectivo de píxeles 20,1 M px

Objetivo zoom 8Xzeiss Vario-Tessar, $\mathrm{f}=4,5 \mathrm{~mm}-36 \mathrm{~mm}$

Imágenes fijas: compatible con JPEG (DCF, Exif, MPF Baseline), compatible con DPOF Películas: MPEG-4 AVC/H264

Memoria interna: $27 \mathrm{MB}$, externa micro SD

Flash: Sensibilidad ISO ajuste automático.

Salidas: USB Hi Speed USB 2.0

Compatibilidad: Windows, Windows vista, Macintosh.

Se utilizó un rotador de polaroid manual, diámetro $45 \mathrm{~mm}$ con apreciación de $\pm 1^{\circ}$ de ángulo acimutal. Durante todas las tomas en cuarto obscuro a $0.1 \mathrm{~lx}$, se mantuvo una temperatura de $25^{\circ}$ y la imagen bajo una retroiluminación constante de una lámpara osram de 50 Watts AC con reflector dicroico [22].

Todos los elementos se sujetaron a un tablero melamínico suspendido sobre capa de esponja suave de 1 pulgada más una capa de plástico de burbujas en configuración sánduche para minimizar la transmisión de posibles vibraciones en la mesa.

En la figura 9 se muestra algunas de las imágenes obtenidas, cada una obedece a un ángulo acimutal característico, se nota la similitud entre el primer cuadro $\left(45^{\circ}\right)$ con el quinto $\left(225^{\circ}\right)$, así también el segundo $\left(90^{\circ}\right)$ con el sexto $\left(270^{\circ}\right)$. 


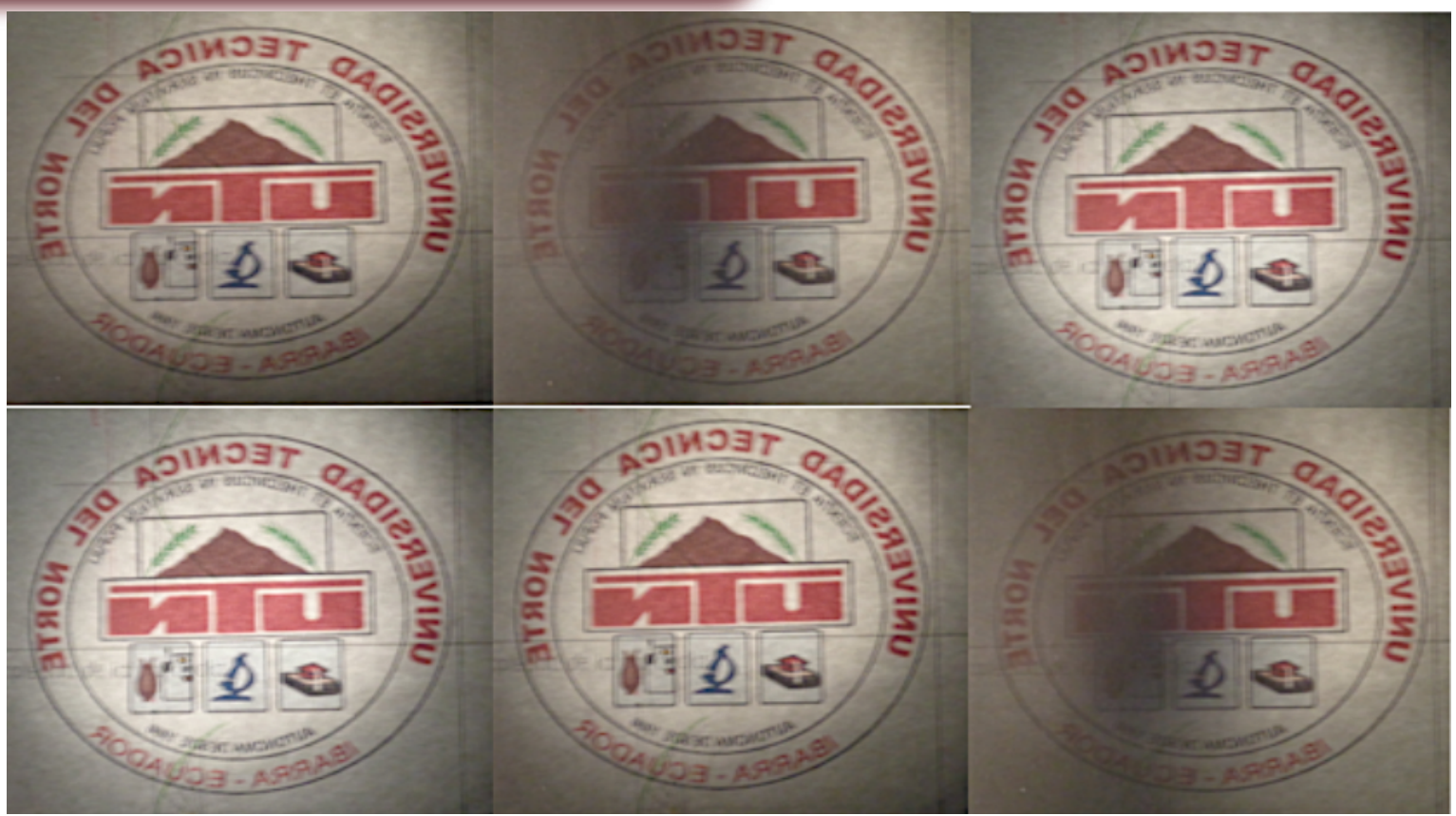

Fig. 9. Imágenes capturadas con polaroide analizador a $45^{\circ}, 90^{\circ}, 135^{\circ}, 180^{\circ}, 225^{\circ}$ y $270^{\circ}$.

\section{Recorte de las imágenes}

En el proceso de recorte de imagen se estableció un punto de referencia para realizar la comparación de la imagen original con la polarizada, considerando que las imágenes polarizadas sufren inversión y un leve desplazamiento debido al grosor del vidrio.

Con este análisis y de acuerdo con la función de MATLAB "imcrop (imagen, [x1, y1, x2, y2])" se estableció un recorte de imagen de $512 \times 512$ pixeles, considerando los puntos de referencias para una imagen polarizado de $\mathrm{x} 1=1000, \mathrm{y} 1=800, \mathrm{x} 2=512$ y y2=512 y para la imagen original los puntos $\mathrm{x} 1=1110$ y $\mathrm{y} 1=740$, los valores de $\mathrm{x} 2$ y y2 se mantienen.

\section{Binarización de la imagen}

El software que mejor se adaptó a las necesidades para el proceso de análisis de imagen es MAT LAB permitiendo fácilmente la binarización [23], segmentación y comparación de las imágenes.

La binarización permite una comparación más eficiente entre imágenes debido a que solo se consideran los valores de 0 y 1 (se cuantizan) por cada pixel analizado, haciéndolo más fácil que trabajar con una imagen en escala de grises o considerando todos los colores RGB.

\section{Segmentación de Imagen por División del cuadro}

Si dividimos la pantalla en $N$ cuadros utilizando la sucesión; $N=2^{2 x n}$, con $n=1,2,3$, $4, \ldots . . \quad(20)$ 
así por ejemplo la primera partición con $n=1$, nos da: $N=2^{2 \times 1}=4 \quad$ cuatro partes como se indica a continuación en la figura 10 a):

a)

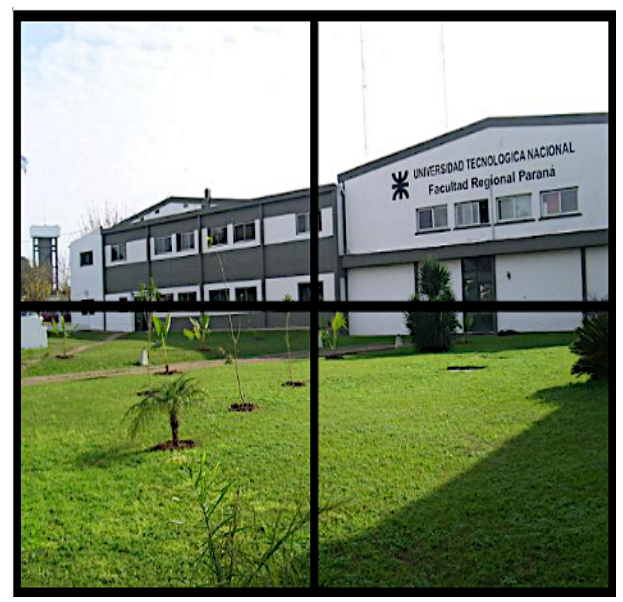

b)

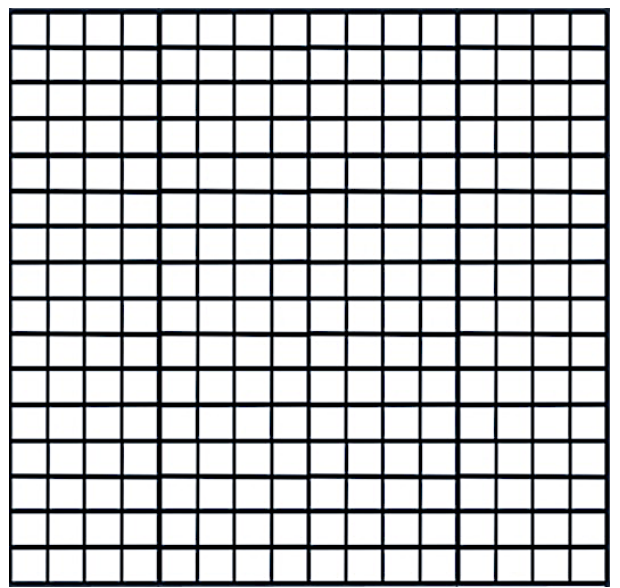

Fig.10. a) Segmentación de una imagen en su primera partición, $n=1$, b) Segmentación de una imagen en su cuarta partición, $n=4$.

Entonces podremos analizar la imagen por subcuadros, en busca de semejanzas y diferencias entre ellos, estas diferencias pueden ser: el número de pixeles rojos (R), pixeles verdes $(G)$ y pixeles azules $(B)$, igualmente pueden incorporarse otras multiples características como repeticiones por filas o por columnas etc. El resultado de esta búsqueda será la elección celdas únicas e inconfundibles, si dos celdas tienen características similares, se consideraran "confusamente iguales" y no serán únicas.

Mientras más grande la celda $\mathrm{S}$ tendremos más pixeles por analizar, reduciremos el tamaño de las celdas a la cuarta parte, en compensación debemos repetir el proceso 4 veces, es necesario una subdivisión que contenga un número representativo de pixeles que haga a la subcelda única y diferente de las otras.

Siguiendo la sucesión: $N_{n}=2^{2 x n}=4^{n}$, para $\mathrm{n}=4$ tendremos $16 \times 16=256$ cuadros, fig. 10b)

$N_{2}=2^{2 x 4}=256$ cuadros con $\quad \frac{800 \times 1200 \text { pixeles }}{256}=3750 \mathrm{px}$

\section{Comparación}

Se toma los resultados de $n=4$, es decir la segmentación de 256 fig. 10b) y en ella se realiza el proceso de comparación fragmento por fragmento del cuadro, para la observación de cambios más específicos en las imágenes analizadas bajo un mismo umbral para los tonos de grises y se contabiliza el número de coincidencias y número de cambios respecto al original. Se desecha la comparación por colores debido al tamaño de la tarea. 


\section{RESULTADOS}

Se expone el patrón de cambios de superación del umbral binarizado de tonos de gris para cada fragmento con el polaroide analizador rotado un ángulo acimutal $\boldsymbol{\theta}$. Los subfotogramas que se expone no son tablas ni figuras, no obstante, se los llama figuras para no perder el orden en la numeración.

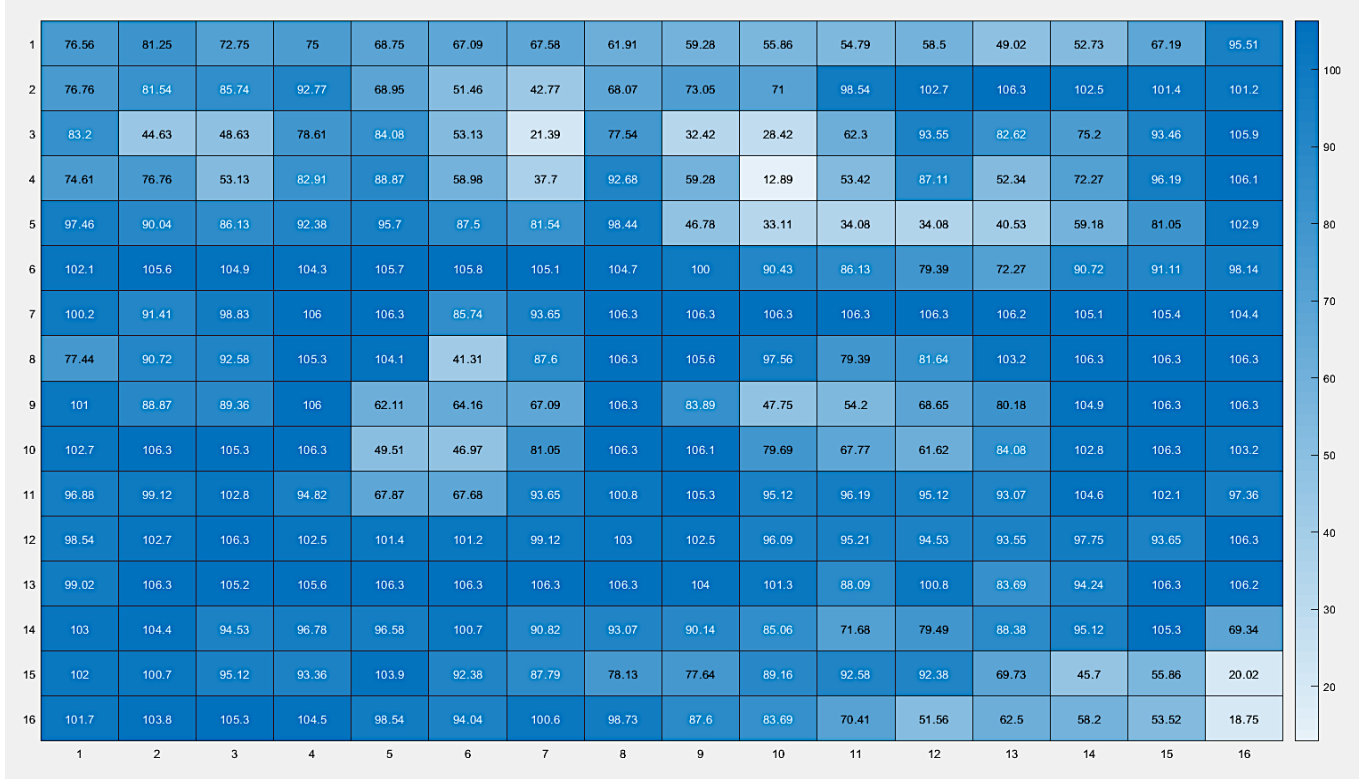

Fig.11. Original vs Angulo $0^{\circ}$

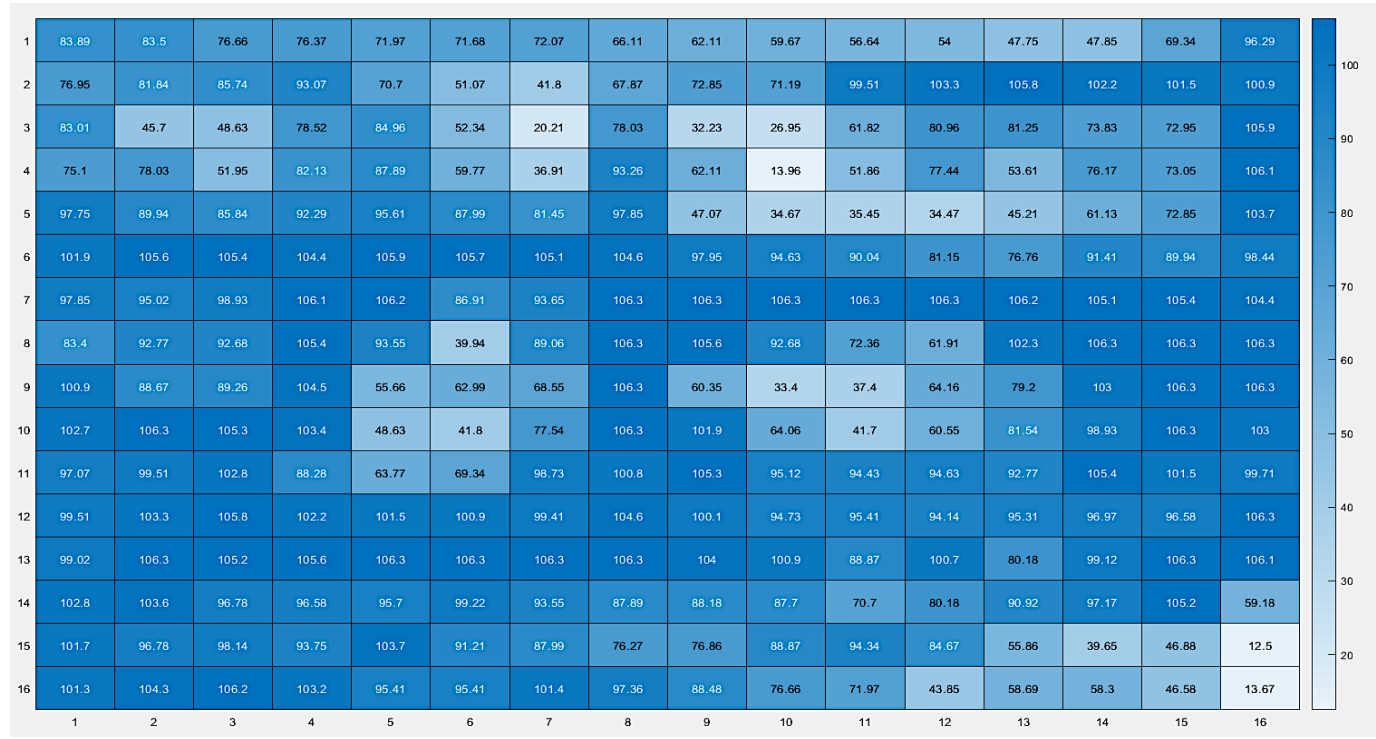

Fig.12. Original vs Angulo $180^{\circ}$ 
Se observa la identidad de la figura $\theta=180^{\circ}$ con el patrón obtenido cuando $\theta=0^{\circ}$, la abundante presencia de cuadrados azules confirma la concordancia del eje del polaroid analizador con el plano de polarización de los rayos provenientes de la imagen reflejada.

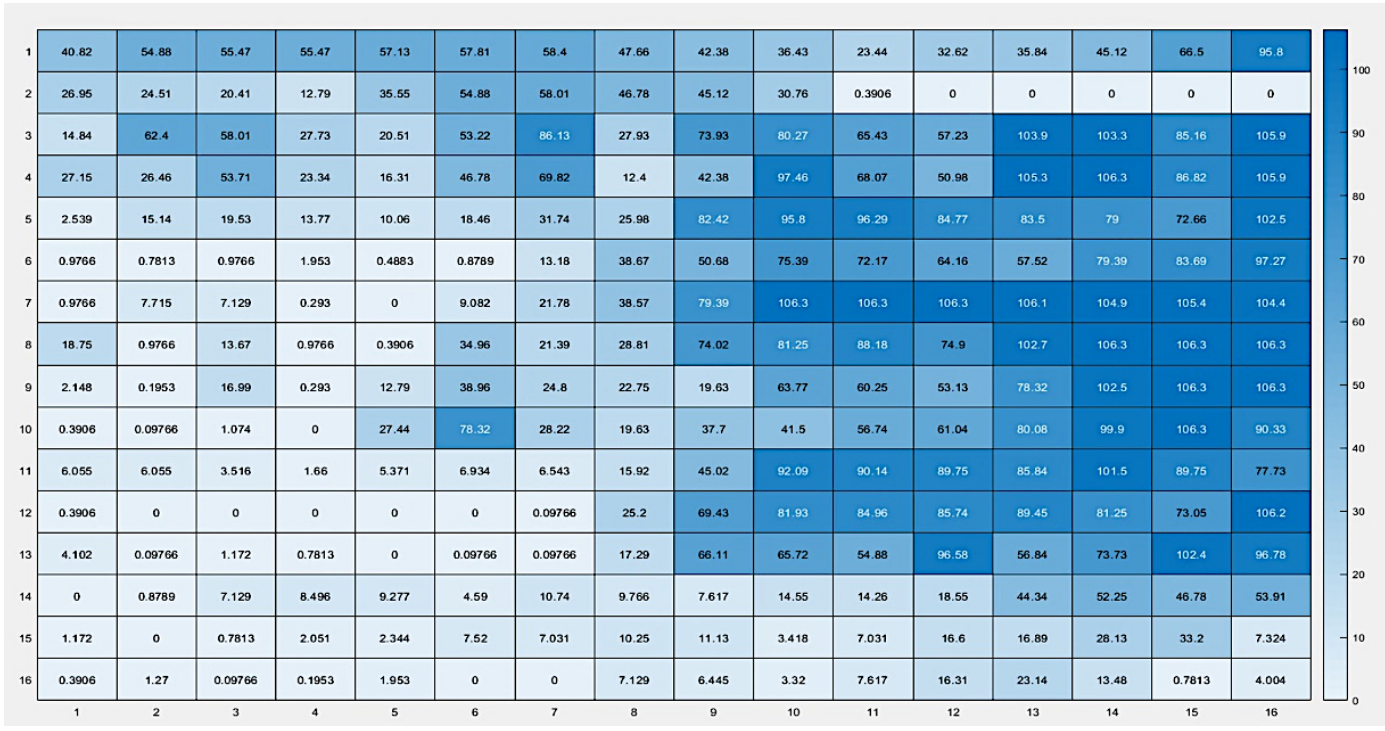

Fig.13. Original vs Angulo $90^{\circ}$

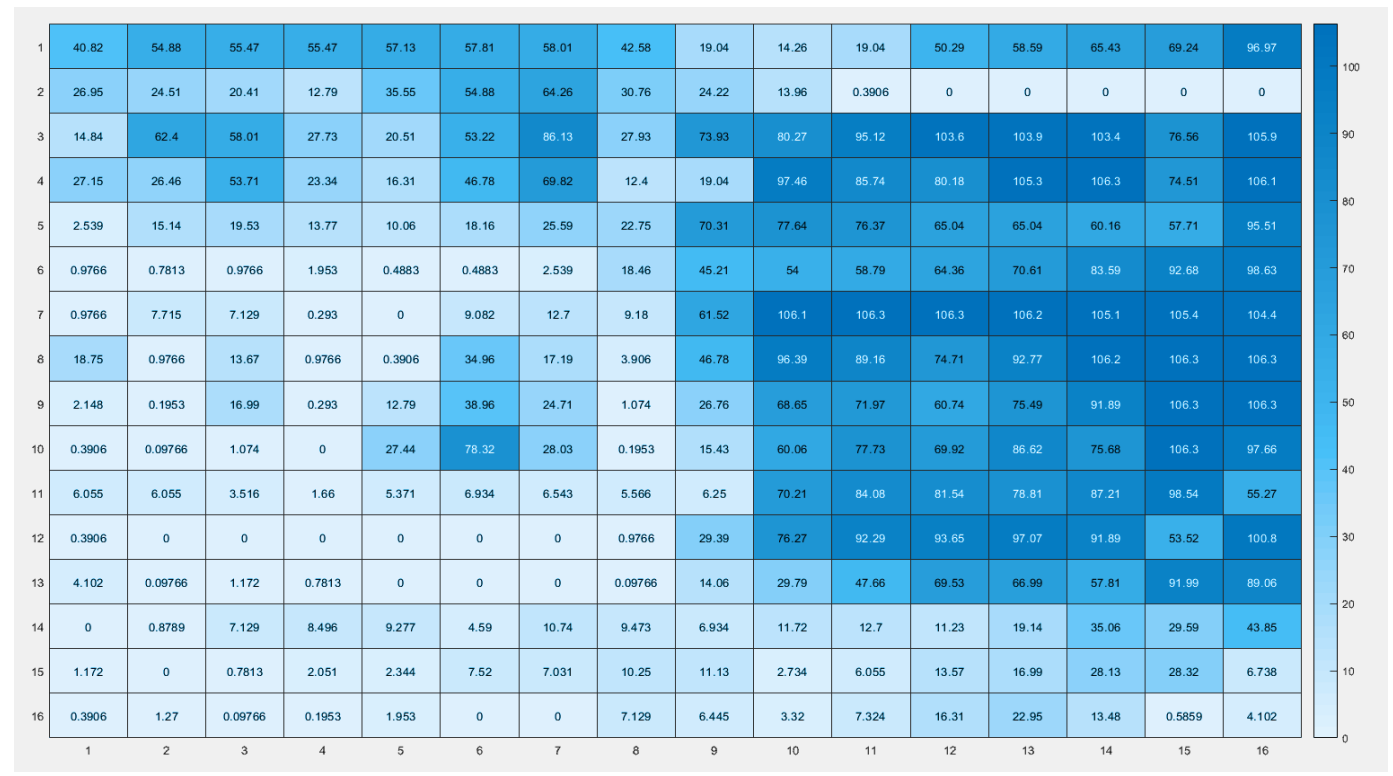

Fig.14. Original vs Angulo $270^{\circ}$

La similitud entre esta tabla y la obtenida a $90^{\circ}$ con el oscurecimiento de los subfotogramas en la esquina inferior izquierda lo que evidencia una alta DOP de los rayos reflejados debido a las leyes de Brewster y Malus. Esto nos indica que es un efecto de la polarización lineal durante el reflejo. 
Los ángulos acimutales de menor transparencia son los de $90^{\circ}$ y $270^{\circ}$ grados, de acuerdo con la figura $7 \mathrm{~b}$ podemos concluir que se trata de polarización lineal con onda vertical (fórmula 4) representada por el vector; $(-1,0,0)$ en la esfera de Poincaré. Éste es el aporte científico de la presente investigación.

\section{DISCUSIÓN Y CONCLUSIONES}

\section{Discusión}

Si se elige evaluar cada sector o fragmento con Vectores de Stokes como en la fórmula (15), identificando también la matriz de transformación de Mueller (fórmula 17) para el sistema, se podrá encontrar el nuevo vector del fragmento como producto matricial de la fórmula (18) y así predecir su intensidad. El número de cálculos crecerá con el número de fragmentos de acuerdo con la fórmula (20) y además se debe obtener las imágenes experimentalmente, el tamaño de la tarea supera en mucho a la presente investigación. Se escogió el método de umbral por su ventaja de que el cálculo es más simple que otros y la velocidad de operación es más rápida, esto dentro de una segmentación regular condicionada por una dependencia cartesiana del grado de polarización.

\section{Conclusiones}

Los ángulos acimutales de menor transparencia son los de $90^{\circ}$ y $270^{\circ}$ grados, de acuerdo a la figura $7 \mathrm{~b}$ podemos concluir que se trata de polarización lineal con onda vertical (fórmula 4) representada por el vector; $(-1,0,0)$ en la esfera de Poincaré. Este es el aporte científico de la presente investigación.

En el caso de captación de imágenes reflejadas en dieléctricos de objetos cercanos siempre aparecerá un patrón de "mancha oscura", de acuerdo con los subfotogramas, obedecen a la polarización lineal en ángulos cercanos al ángulo de Brewster para el dieléctrico y constituyen un limitante para la resolución y la nitidez.

El patrón de "mancha oscura" de la reflexión desaparece al momento de utilizar espejo metalizado, esto demuestra el carácter ondulatorio del fenómeno.

El uso de doble cámara podría ser una solución interesante al momento de complementar imágenes en el sector de la mencionada "mancha oscura" o sombra.

Es posible caracterizar el estado de polarización de cada cuadro de una segmentación con algún vector de Stokes, su transformación tras cualquier tipo de polarización se puede evaluar con una matriz de transformación de Moeller. El patrón de cambio de una imagen binarizada y regularmente segmentada en forma $n \times n$, se caracterizará por el mismo número $\mathrm{n} \times \mathrm{n}$ de matrices de Moeller 4x4, una para cada subcuadro, es decir con un tensor. 
Para evaluar el patrón de cambio por colores, en futuras investigaciones, se recomienda utilizar una imagen regularizada con $\mathrm{n} x \mathrm{n}$ colores y el mismo número de matrices de Moeller para la búsqueda del tensor de transformación.

La herramienta de Mat Lab cumple con las expectativas de procesamiento de imagen reflejada en dieléctricos. Para futuras investigaciones se recomienda una herramienta más automatizada en honor al tiempo.

\section{BIBLIOGRAFIA}

E. Hecht: OPTICS, 5.7 Los sistemas ópticos, pp. 151-233 , 2002, San francisco, New York, Boston,..., Addison Wesley.

A.N. Zaidel Y.I.; Ostrovsky, G. V. O. : TÉCNICA Y PRÁCTICA DE ESPECTROSCOPÍA. Iluminación y Enfoque de Aparatos Espectrales, pp. 181-217, 1979, Moscú, Ed. Mir.

Jain A.; Anees, P.; Tamang, R.; Pendyala, N.; \& Banerjee, A.: Development of Electrooptical Characterization Test Bench for High Performance Infrared Focal Plane Area Array Detectors (Lcc).

Christian Buil: CCD Astronomy, 4 Image Procesing, 1991, Richmond Virginia ,pp153211, Willmann-Bell, Inc.

D. B. Sibujin: Curso de Física General, tomo 4«Elementos Polarizadores, pp. 264-272, 1985, Moscú, Nauka.

G. Anna; F. Goudail ; D. Dolfi: «Polarimetric target detection in the presence of spatially fluctuating Mueller matrices.,» Optics Letters, vol. 36, n²3, 2011, pp. 1-4.

Chipman, R: Handbook of Optics, Polarimetry, p. 22.1-22.23, 1995, M. G. Hill (Ed.), (Second,). New York.

Z. B. Begunov: TEORÍA DE SISTEMAS ÓPTICOS, Óptica de rayos paraxiales, pp. 77-91, 1976, Moscú, Ed. Mir.

Vargas, G.; Neira, O.; Arango, R.; Fernando, D.; Métodos de segmentación de nubes en imágenes satelitales, Cloud segmentation methods applied to satellite images. Tecnura, 17(36), 96-110, 2013.

Guoxiu Huang; Shoichiro Oda; Yuichi Akiyama; Hiroyuki Irie; Setsuo Yoshida; Hisao Nakashima, T. H.: Poincaré Sphere. Fujitsi Limited, 1-2-3. Retrieved from Fujitsu

E. Hecht: OPTICS, 5.7 Polarización pp. 324-377, (2005, San francisco, New York, Boston,..., Addison Wesley Tercera Ed. Madrid, Pearson.

B. M. Yavorski; A. A. Detlaf: PRONTUARIO DE FÍSICA, Ondas electromagnéticas, pp. 416-434, 1985, Moscú, Ed.Mir. 
J. M. Bennet: Handbook oof OPTICS, 2.- Fresnel equations, pp. 5-4 5-12, 1995, de, New York, San Francisco y otras, Mc GRAW-HILL,INC.

Guoxiu Huang; Shoichiro Oda; Yuichi Akiyama; Hiroyuki Irie; Setsuo Yoshida; Hisao Nakashima: Poincaré Sphere. Fujitsi Limited, 1-2-3. Retrieved from Fujitsu

Progreso, F.. Manejo e interpretación polarimétrica de las matrices de Mueller, 55(3), 201-210, 2009

Luna, R. Espinoza; S. Hinojosa Ruiz; G. A. R. (n.d.): Manejo e interpretación polarimétrica de las matrices de Mueller. REVISTA MEXICANA DE FÍSICA, 55.

Son, T. V.; Truong, V. V.; Do, P. A.; Haché, A.: (2016). Ultra-thin, single-layer polarization rotator. AIP Advances, 6(8). https://doi.org/10.1063/1.4960552

P. Wang; Q Chen; G. Q. W. R. K. Gu: Polarimetric Image Discrimination with Depolarization Mueller Matrix, IEEE Photonics Journal, vol. 8, nº, pp. 1-14, 2016.

E. Hecht: Optics, «8.13 A Matemátical Description of polarization, pp. 374-379, 2002, New York, Boston, San Francisco..., Addison Wesley.

D. B. Sibujin, Curso de Física General, T4 Análisis de la luz polarizada, pp. 472-480, 1985, Moscú, Ed. Nauka.

S. Yuheng; Y. Hao: «Image Segmentation Algorithms Overview,» vol. 1, pp. 1-6, 2017.

A. N. Zaidel; Y. I. Ostrovsky ; G. V. Ostrovskaya,: TÉCNICA Y PRÁCTICA DE ESPECTROSCOPÍA, Enfoque del aparato espectral, pp. 207-217, Moscú, 1979, Ed. Mir.

Vargas, G.; Neira, O.; Arango, R.; Fernando, D.:. Métodos de segmentación de nubes en imágenes satelitales Cloud segmentation methods applied to satellite images. Tecnura, 17(36), 96-110, 2013. 